\title{
Anaplastic meningioma: octreotide therapy for a case of recurrent and progressive intracranial disease
}

\author{
Richard Rammo, MD, ${ }^{1}$ Adam Rock, BS, ${ }^{2}$ Andrea Transou, BS, ${ }^{1}$ Aditya Raghunathan, $M D,{ }^{3}$ and \\ Jack Rock, MD ${ }^{1}$
}

\begin{abstract}
1'Department of Neurosurgery, Hermelin Brain Tumor Center, Henry Ford Hospital, Detroit, Michigan; ${ }^{2}$ Rosalind Franklin University Medical School, North Chicago, Illinois; and ${ }^{3}$ Division of Anatomic Pathology, Mayo Clinic, Rochester, Minnesota
\end{abstract}

\begin{abstract}
Meningiomas are common intracranial tumors categorized as Grades I-III per the current WHO guidelines. A small percentage of meningiomas are Grades II and III, which are likely to recur after initial treatment. Grade III meningiomas are considered to be malignant and warrant aggressive management. If surgery and radiation fail to produce lasting remission, effective treatment options for patients with progressive anaplastic meningiomas are elusive. The authors present the case of a patient with a meningioma that gradually progressed from Grade I to Grade III over 12 years despite repeated surgery and radiation therapy. The patient has been in remission for over 3 years following octreotide therapy. http://thejns.org/doi/abs/10.3171/2015.1.JNS142260
\end{abstract}

KEY WORDS intracranial; meningioma; octreotide; oncology

$\mathrm{M}$ Eningiomas are common intracranial tumors categorized as Grades I-III by the WHO. ${ }^{11}$ The majority of Grade I meningiomas behave in an indolent manner and can be completely removed by resective surgery, or their growth can be controlled by radiation therapy. A small percentage of meningiomas are Grades II and III, which have variable growth patterns and are likely to recur after initial treatment. Grade III meningiomas are considered to be anaplastic (malignant) and warrant aggressive management. Unfortunately, there are limited treatment options beyond surgery and radiation for patients with Grades II and III meningiomas. In many patients the Grade II meningioma will gradually convert to Grade III. Initial treatment(s) will fail in most patients with Grade III meningiomas, and they will ultimately succumb to recurrent and progressive tumor. In this report, we present an encouraging case of a patient who-after 5 intracranial surgeries and 3 stereotactic radiation treatments for malignant meningioma-has had stable disease for 3.5 years following the initiation of octreotide therapy. We also review the current treatment options for recurrent malignant meningioma.

\section{Case Report}

History and Examination

In 1994, a 35-year-old woman presented with worsening seizures and was found to have a right frontal mass, which was embolized and subsequently resected. Histological examination demonstrated a proliferation of relatively uniform, bland meningothelial cells that were frequently arranged as the characteristic whorls (Fig. 1). At most, we identified 2 mitoses per $10 \mathrm{hpf}$ (magnification $\times 400$ ). A thin rim of brain tissue was present and did not show invasion by the meningioma. Per the 2007 WHO Classification of Tumours of the Central Nervous System guidelines, ${ }^{11}$ this tumor was consistent with the diagnosis of a Grade I meningioma. In 1998, the patient experienced a small recurrence that was treated by Gamma Knife radiosurgery with 16 Gy to the $90 \%$ isodose line. Subsequent progression at the treated site led to additional radiosurgery in 2002 (16 Gy to the 15\% isodense line) and 2004 (15 Gy to the $40 \%$ isodense line).

In 2008, the tumor continued to progress and was noted primarily on the right side of the falx and extending slightly across the midline, occluding the sagittal sinus (Fig. 2A). The next intervention was embolization followed by resection leading to minimal residual tumor at the posterior aspect of the involved sagittal sinus (Fig. 2B). Histological examination revealed an atypical meningioma (WHO Grade II; Fig. 2C) with marked cytological atypia, up to 6 mitoses per $10 \mathrm{hpf}$, and a Ki 67 labeling index of approximately $30 \%$ in the most active areas. Repeat MRI 2 months postoperatively demonstrated an increased size of the residual tumor. Stereotactic radiation was recom-

ABBREVIATIONS PBS = phosphate-buffered saline; SST = somatostatin receptor.

SUBMITTED October 1, 2014. ACCEPTED January 28, 2015.

INCLUDE WHEN CITING Published online August 14, 2015; DOI: 10.3171/2015.1.JNS142260.

DISCLOSURE The authors report no conflict of interest concerning the materials or methods used in this study or the findings specified in this paper. 


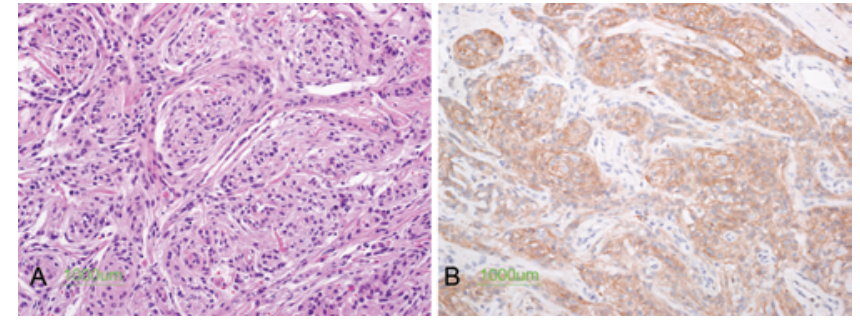

FIG. 1. A: On histological examination, the tumor showed a proliferation of relatively uniform, bland meningothelial cells that were frequently arranged as characteristic whorls and with, at most, 2 mitoses per 10 hpf. WHO Grade I. H \& E. B: The octreotide immunohistochemical stain showed moderately strong membranous and granular cytoplasmic staining in a majority of the tumor. um $=\mu \mathrm{m}$. Figure is available in color online only.

mended but the patient declined. Seven months after the last surgery, the patient presented to the emergency room with left-sided weakness, headache, and blurry vision, and MRI revealed a progressive enlarging meningioma with surrounding edema (Fig. 3A). In the absence of effective treatment options and in the presence of significant cerebral mass effect, reoperation followed, as did histological examination, which demonstrated an anaplastic (malignant) meningioma (WHO Grade III; Fig. 3B) with cytological anaplasia, up to 24 mitoses per $10 \mathrm{hpf}$, and a Ki 67 labeling index of up to approximately $75 \%-80 \%$ in the most active areas. Radiation was again discussed, but again the patient refused.

Magnetic resonance imaging in early 2009 showed the residual meningioma increased in size over the right frontal region and an enhancing intramuscular lesion within the temporalis muscle (Fig. 4A). Surgery was undertaken, and the histological sections revealed residual anaplastic meningioma (WHO Grade III) involving skeletal muscle, with frequent mitoses, an overall Ki 67 labeling index of approximately $35 \%$, and focal areas of up to about $75 \%$. Five months later, a new 2-cm lesion was noted along the right frontal dura mater, and alternatives to surgery were discussed extensively in the tumor board.

\section{Octreotide Treatment and Surgery}

Given citations in the literature indicating the occasional beneficial effects of octreotide, the patient consulted with the hematology/oncology department. Scintigraphy confirmed octreotide uptake in the right frontal region (Fig. 4B). She was started on octreotide acetate therapy (Sandostatin LAR, Novartis Pharmaceuticals Corp.), 30 mg once every 4 weeks. In early 2010, follow-up MRI revealed interval development of a bilobed mass in the extracranial right frontal subcutaneous tissues with central necrosis (Fig. 4C). She again opted for surgery, and the mass was found to be residual anaplastic meningioma (WHO Grade III).

Postoperatively, the patient was restarted on octreotide therapy, but the frequency was increased to once every 3 weeks for 4 months before later resuming once every 4 weeks in 2011, at the patient's request. Repeat MRI demonstrated a $1.5-\mathrm{cm}$ enhancing lesion in the right frontal region (Fig. 4D). Octreotide therapy was increased to 40
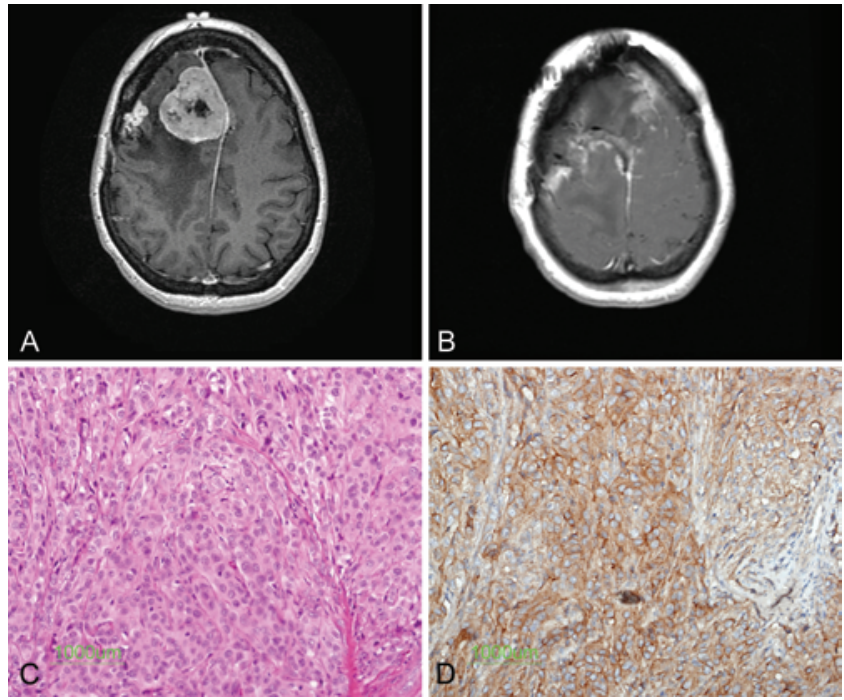

FIG. 2. A: Preoperative axial T1-weighted MR image with contrast demonstrating tumor recurrence. B: Postoperative axial T1-weighted MR image demonstrating nearly complete resection with only residual tumor noted at the anterior border of the resected sagittal sinus. C: The meningothelial proliferation is predominantly arranged as solid sheets, with an inconspicuous whorl formation. The neoplastic cells show cytological atypia, with higher nuclear/cytoplasmic ratios, pleomorphic nuclei, hyperchromasia, and prominent nucleoli. Up to 6 mitoses were identified per $10 \mathrm{hpf}$, corresponding to atypical meningioma, (2007) WHO Grade II. H \& E. D: The octreotide immunohistochemical stain in this material also shows moderately strong membranous and granular cytoplasmic staining in a majority of the tumor. Figure is available in color online only.

mg every 4 weeks. Since mid 2011, the patient has continued with this regimen and her disease has remained stable as of July 2014 with regression of the right frontal lesion noted in 2011 and without progression or recurrence at any other site (Fig. 5). She has taken no other medications (that is, vitamins, herbal treatments, or other orally ingested agents that might be considered alternative treatments).

\section{Histological Analysis of Octreotide Expression}

Following this remission-free period, histological sections from the surgically resected specimens were reviewed and examined for the expression of octreotide. Briefly, 5- $\mu \mathrm{g}$-thick sections from archival formalin-fixed, paraffin-embedded tissue from surgeries performed in 1994, early 2008, and late 2008 were deparaffinized, rehydrated, and stained progressively with Biocare Medical's CAT hematoxylin for 1 minute. The slides were then counterstained with Richard-Allan Scientific's Eosin-Y for 1 minute, dehydrated, and cleared, and a coverslip was added. There are 6 subtypes of octreotide receptors: 1, 2A, 2B, 3, 4, and 5. Immunohistochemical staining was performed to evaluate expression of the octreotide receptor subtype $2 \mathrm{~A}$ antibody as a surrogate for octreotide expression. The sections were pretreated with formic acid (80\% solution for 8 minutes) and subsequently immersed in a $0.3 \%$ hydrogen peroxide solution diluted in phosphate-buffered saline (PBS) for 30 minutes to neutralize endogenous peroxide activity. Sections were incubated overnight at $4^{\circ} \mathrm{C}$ with a monoclonal antibody against 

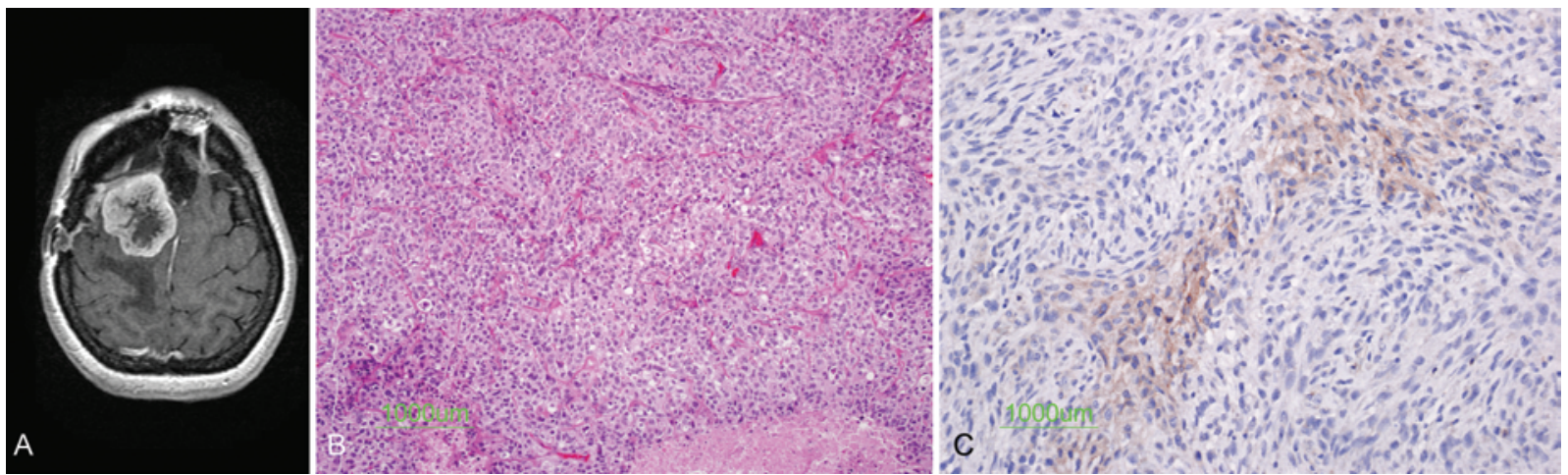

FIG. 3. A: Preoperative axial T1-weighted MR image with contrast demonstrating a large recurrence in the right frontal lobe. B: The meningothelial proliferation is predominantly arranged as sheets, shows marked cytological anaplasia, and has up to 24 mitotic figures per $10 \mathrm{hpf}$, corresponding to anaplastic meningioma, (2007) WHO Grade III. H \& E. C: The octreotide immunohistochemical stain in this material shows weak membranous and cytoplasmic staining in focal areas. Figure is available in color online only.

the octreotide receptor 2A (clone PA3-109, 1:5000 dilution, Thermo Fisher Scientific) diluted in a PBS solution containing $2 \%$ horse serum, $0.3 \%$ Triton X-100, and $0.1 \%$ sodium azide. Sections were then incubated with an antimouse biotinylated IgG antibody (Biocare Medical) for 1 hour, and the antibody complex was revealed using the avidin biotin peroxidase method (4plus detection system, Biocare Medical) with horseradish peroxidase and with diaminobenzidine as a substrate. All sections were lightly counterstained with Mayer's hematoxylin.

\section{Discussion}

While not all intracranial meningiomas require treatment and many can be monitored with MRI for growth, generally, the first-line treatment is surgery, which for many patients represents a definitive and curative option. However, meningiomas on the skull base and along the venous sinuses are often not amenable to complete excision and can recur. In some patients radiotherapy may be an initial treatment option, but more often it is used as an adjuvant or secondary therapy. ${ }^{9}$

Various chemotherapeutic agents have been used in patients with recurrent meningiomas, including hydroxyurea, temozolomide, irinotecan, tamoxifen, and mifepristone (RU-486). To date, none of these agents has shown consistent efficacy in controlling the growth of recurrent meningiomas. ${ }^{3,6,7,10,12,17}$

Interferon alpha-2B is thought to exert both antiproliferative and antiangiogenic effects that have been implicated in the treatment of meningiomas. The efficacy of subcutaneous interferon alpha-2B in the treatment of these tumors was investigated in 6 patients exhibiting recurrent or unresectable meningioma. ${ }^{10}$ Results demonstrated tumor stabilization in 6 of 6 patients, with tumor regression observed in 1 of 6 patients. The beneficial effects of interferon alpha-2B treatment were noted to last from 6 to 14 months with minimal adverse effects (for example, flu-like symptoms, injection site pain, and mild leukopenia). Interferon alpha-2B may have been effective in the treatment of these patients, and although the investigators suggested that these results warrant follow-up with a larger prospective study, it is unclear whether these positive treatment effects may have been confounded by the natural history of the disease..$^{10}$

Hydroxyurea is hypothesized to prevent tumor progression via inhibition of ribonucleotide reductase and subsequent DNA synthesis because cells undergoing this process may eventually undergo apoptosis in the $\mathrm{S}$ phase of the cell cycle. In a series assessing hydroxyurea treat-
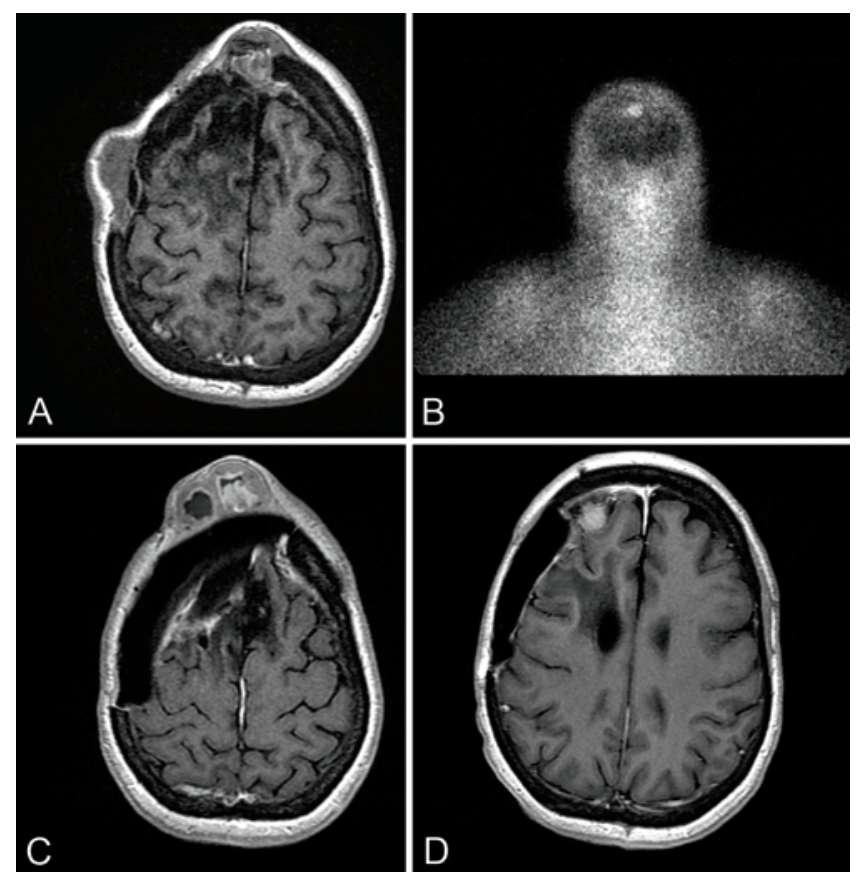

FIG. 4. A: A 2009 preoperative axial T1-weighted MR image with contrast demonstrating extracranial recurrence of the tumor. B: Octreotide scintiscan demonstrating hyperintensity in the frontal region. C: Early 2010 preoperative axial T1-weighted MR image with contrast demonstrating large frontal extracranial recurrence. D: A 2011 axial T1weighted MR image with contrast demonstrating a small recurrence in the right frontal lobe. 


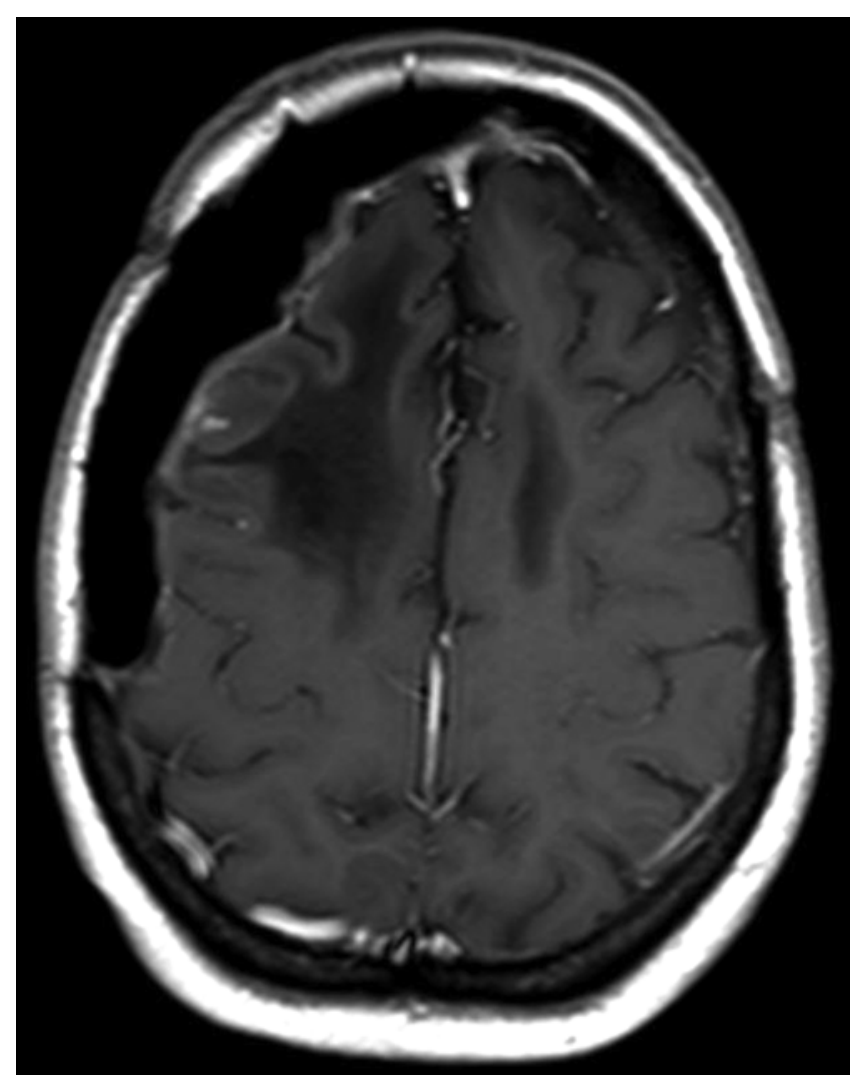

FIG. 5. Surveillance axial T1-weighted MR image with contrast from July 2014 shows regression of the lesion noted in 2011 and no evidence of progression or recurrence in any other location.

ment of atypical and malignant meningiomas, researchers found no significant medical benefit as tumor size progressed on both clinical and neuroimaging evaluations throughout the therapeutic intervention. ${ }^{12}$ In addition, hydroxyurea therapy was associated with a reversible myelosuppression that most frequently involved neutropenia and anemia. While hydroxyurea was determined to have no foreseeable indication in tumor regression or atypical and malignant meningiomas, the authors considered that it might be beneficial in the stabilization of some benign meningiomas.

The efficacy of temozolomide, an alkylating agent, was studied to assess progression-free survival in patients with treatment-resistant recurrent meningiomas. ${ }^{4}$ In this investigation, temozolomide appeared to have no clinical benefit in the treatment of recurrent meningiomas as indicated by the lack of progression-free survival in any patient after 6 months of therapeutic intervention. ${ }^{4}$

In another series researchers conducted a phase II study on the effects of imatinib mesylate, a platelet-derived growth factor receptor inhibitor, on recurrent meningiomas. ${ }^{17}$ Despite the general tolerability of the imatinib mesylate treatment, results failed to show significant activity in recurrent meningiomas. Investigators hypothesized that given the advanced state of disease and significant pretreatment in the patients, molecular alterations could account for the lack of positive effects with imatinib mesyl- ate, which could be more beneficial in less advanced cases of meningioma. ${ }^{17}$

To date, however, none of the agents previously noted as well as others in the literature has been shown to be consistently effective in controlling the growth of recur-

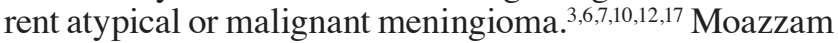
et al. reviewed a spectrum of chemotherapeutic agents and noted this concern, recommending that multidrug therapy could be optimal in the treatment of meningiomas and that identifying cellular receptors and intracellular signaling pathways in a patient-specific manner could have profound effects on tumor stability or even regression. ${ }^{13}$

In our patient the tumor progression continued despite repeated and aggressive surgery and adjuvant stereotactic radiation. Given the lack of demonstrated efficacy noted in the literature, we did not consider chemotherapy a reasonable next step when the tumor progressed.,.9 Additionally, at the last time of progression in 2011, recurrence was limited and asymptomatic and the patient refused further surgery. A thorough review of the literature revealed several studies on the use of octreotide for various tumors including meningioma. Following discussion with the patient, we elected to initiate octreotide therapy.

Octreotide is an inhibitory neuropeptide released by the hypothalamus. ${ }^{9}$ Many brain tumors express octreotide receptors, but meningiomas also frequently demonstrate a high level of expression. ${ }^{2,8}$ The presence of receptors can be confirmed by octreotide scintigraphy or staining of a specimen. ${ }^{2,9}$ Octreotide is thought to be antimitotic, as growth is suppressed. ${ }^{8}$ Schulz et al. had a series of 8 patients with meningiomas, 3 with 2 operations and 2 with 3 operations before octreotide therapy. ${ }^{14}$ Only 1 patient had disease progression, and that occurred 60 months after initiation of octreotide therapy. ${ }^{14}$

While tumor regression is uncommon with octreotide therapy, it was observed in our patient and also in a study by García-Luna et al. ${ }^{5}$ Shrinkage may be attributable to mechanisms external to meningioma receptors, such as inhibition of angiogenesis or reduction in edema. ${ }^{8}$

A study by Johnson et al. demonstrated growth despite treatment with octreotide. ${ }^{9}$ In a sample of 11 patients, only 2 had long-term suppression of disease. Poor response could be related to the aggressive nature of the meningiomas in this group (that is, 5 with anaplastic meningiomas and 2 with atypical meningiomas). Chamberlain et al. had more promising results, with $44 \%$ of patients in a group of 16 demonstrating disease-free progression at 6 months. ${ }^{2}$

Ironically, some in vitro studies have demonstrated inverse effects of octreotide, leading to an increased size of meningiomas after treatment. ${ }^{2}$

The spectrum of octreotide effects in the treatment of recurrent meningiomas in the various studies begs the question, is this an effect at the receptor level? $?^{2,15}$ Possibilities include receptor inactivity and lack of octreotide affinity for specific receptors. ${ }^{2}$ Five subtypes of octreotide have been identified (somatostatin receptor $[\mathrm{SST}]_{1-5}$ ) with 2 isoforms of $\mathrm{SST}_{2}\left(\mathrm{SST}_{2 \mathrm{~A}}\right.$ and $\left.\mathrm{SST}_{2 \mathrm{~B}}\right) \cdot{ }^{15}$ The presence of different subtypes and the use of synthetic octreotide has certain implications.$^{15}$ The first may relate to the drug's affinity for the receptors. $\mathrm{SST}_{2}, \mathrm{SST}_{3}$, and $\mathrm{SST}_{5}$ have higher binding of analogs.${ }^{15}$ The next variable may be the differ- 
ent intracellular pathways thought to be required for the positive clinical effects of octreotide. ${ }^{15} \mathrm{SST}_{2}$ has antiproliferative effects using tyrosine pathways, while $\mathrm{SST}_{3}$ is antiapoptotic through p53.,16 Finally, the quantity of octreotide receptors could affect response, with low numbers of receptors causing a decrease in hormonal effects and high numbers causing antiproliferative effects. ${ }^{15}$ Therefore, the varied outcomes noted in patients undergoing octreotide therapy may be related to several possible mechanisms that are still unclear.

\section{Conclusions}

Our patient's prolonged remission suggests that the use of octreotide has had an impact over the last 3.5 years in delaying the recurrence of meningioma-the longest interval with no growth since 2002. Given the apparent effects of octreotide in our case, it is possible that the tumor expresses the necessary receptor subtype required for octreotide to be clinically effective. However, the encouraging outcome could also be the result of the additive effect of multiple prior therapies, but we believe that is unlikely given the time between treatments. Although the results in our patient are encouraging, further clinical, radiographic, and laboratory follow-up are warranted.

\section{References}

1. Buscail L, Delesque N, Estève JP, Saint-Laurent N, Prats H, Clerc P, et al: Stimulation of tyrosine phosphatase and inhibition of cell proliferation by somatostatin analogues: mediation by human somatostatin receptor subtypes SSTR1 and SSTR2. Proc Natl Acad Sci U S A 91:2315-2319, 1994

2. Chamberlain MC, Glantz MJ, Fadul CE: Recurrent meningioma: salvage therapy with long-acting somatostatin analogue. Neurology 69:969-973, 2007

3. Chamberlain MC, Tsao-Wei DD, Groshen S: Salvage chemotherapy with CPT-11 for recurrent meningioma. J Neurooncol 78:271-276, 2006

4. Chamberlain MC, Tsao-Wei D, Groshen S: Temozolamide for the treatment of resistant recurrent meningioma. Neurology 62:1210-1212, 2004

5. García-Luna PP, Relimpio F, Pumar A, Pereira JL, Leal-Cerro A, Trujillo F, et al: Clinical use of octreotide in unresectable meningiomas. A report of three cases. J Neurosurg Sci 37:237-241, 1993

6. Grunberg SM, Weiss MH: Lack of efficacy of megestrol acetate in the treatment of unresectable meningioma. J Neurooncol 8:61-65, 1990

7. Grunberg SM, Weiss MH, Spitz IM, Ahmadi J, Sadun A, Russell CA, et al: Treatment of unresectable meningiomas with the antiprogesterone agent mifepristone. J Neurosurg 74:861-866, 1991
8. Jaffrain-Rea ML, Minniti G, Santoro A, Bastianello S, Tamburrano G, Gulino A, et al: Visual improvement during octreotide therapy in a case of episellar meningioma. Clin Neurol Neurosurg 100:40-43, 1998

9. Johnson DR, Kimmel DW, Burch PA, Cascino TL, Giannini $\mathrm{C}, \mathrm{Wu} \mathrm{W}$, et al: Phase II study of subcutaneous octreotide in adults with recurrent or progressive meningioma and meningeal hemangiopericytoma. Neuro Oncol 13:530-535, 2011

10. Kaba SE, DeMonte F, Bruner JM, Kyritsis AP, Jaeckle KA, Levin V, et al: The treatment of recurrent unresectable and malignant meningiomas with interferon alpha-2B. Neurosurgery 40:271-275, 1997

11. Louis DN, Ohgaki H, Wiestler OD, Cavenee WK, Burger PC, Jouvet A, et al: The 2007 WHO classification of tumours of the central nervous system. Acta Neuropathol 114:97-109, 2007

12. Mason WP, Gentili F, Macdonald DR, Hariharan S, Cruz CR, Abrey LE: Stabilization of disease progression by hydroxyurea in patients with recurrent or unresectable meningioma. J Neurosurg 97:341-346, 2002

13. Moazzam AA, Wagle N, Zada G: Recent developments in chemotherapy for meningiomas: a review. Neurosurg Focus 35(6):E18, 2013

14. Schulz C, Mathieu R, Kunz U, Mauer UM: Treatment of unresectable skull base meningiomas with somatostatin analogs. Neurosurg Focus 30(5):E11, 2011

15. Schulz S, Pauli SU, Schulz S, Händel M, Dietzmann K, Firsching R, et al: Immunohistochemical determination of five somatostatin receptors in meningioma reveals frequent overexpression of somatostatin receptor subtype sst2A. Clin Cancer Res 6:1865-1874, 2000

16. Sharma K, Patel YC, Srikant CB: Subtype-selective induction of wild-type p53 and apoptosis, but not cell cycle arrest, by human somatostatin receptor 3. Mol Endocrinol 10:1688-1696, 1996

17. Wen PY, Yung WKA, Lamborn K, Dahia PL, Wang Y, Peng $\mathrm{B}$, et al: Phase II study of imatinib mesylate for recurrent meningiomas (North American Brain Tumor Consortium study 01-08). Neuro Oncol 11:853-860, 2009

\section{Author Contributions}

Conception and design: J Rock. Acquisition of data: Rammo, A Rock, Transou, Raghunathan. Analysis and interpretation of data: J Rock, Rammo, Transou, Raghunathan. Drafting the article: J Rock, Rammo, A Rock, Raghunathan. Critically revising the article: J Rock, Raghunathan. Reviewed submitted version of manuscript: J Rock, Raghunathan. Approved the final version of the manuscript on behalf of all authors: J Rock. Study supervision: J Rock.

\section{Correspondence}

Jack Rock, Department of Neurosurgery, Henry Ford Health System, 2799 W. Grand Blvd., Detroit, MI 48202. email: jrock1@hfhs.org. 